Research Article

\title{
The Study of Large-Scale Fading Using a Wavelet Transform Method
}

\author{
Guizhen Lu $\mathbb{D}^{1},{ }^{1}$ Zhi Cao $\mathbb{D}^{1},{ }^{1,2}$ Xingning Jia $\mathbb{D}^{1},{ }^{1}$ and Jingjing Liang ${ }^{1}$ \\ ${ }^{1}$ School of Information Engineering, Communication University of China, Beijing, China \\ ${ }^{2}$ Academy of Broadcasting Planning SARFT, Beijing, China \\ Correspondence should be addressed to Guizhen Lu; luguizhen@cuc.edu.cn
}

Received 27 June 2018; Accepted 30 September 2018; Published 4 December 2018

Academic Editor: Stefania Bonafoni

Copyright (C) 2018 Guizhen Lu et al. This is an open access article distributed under the Creative Commons Attribution License, which permits unrestricted use, distribution, and reproduction in any medium, provided the original work is properly cited.

\begin{abstract}
A wavelet method is proposed to evaluate the modeling of the first- and second-order statistics of large-scale fading from the signal strength measurement. The selection of wavelet is very important in using a wavelet method, and the steps of the wavelet method are given for the study of wave propagation loss. The path loss of measurement is analysed with different levels of wavelet decomposition and compared with an optimized Hata model. The correlation of slow fading on different scales shows that the correlation distance is related to the spatial scale.
\end{abstract}

\section{Introduction}

Modern communication systems must be carefully designed, operated, and optimized in order to satisfy user expectations. The study of electromagnetic wave propagation is of significant importance in the planning and evaluation of wireless communication systems and data networks. The fading in wireless channels plays an important role in the wireless communication system design. This is particularly true in allocating resources and selecting a suitable transmission policy to match the instantaneous fading channel condition $[1,2]$.

The large-scale propagation models give results as path loss versus range. Theoretical and experimental propagation models indicate that the average received signal power decreases logarithmically with distance. A semiempirical method has been proposed in ITU-R P.1546 recommendation for reliable prediction of radio propagation at VHF and UHF bands [3]. The simplest empirical model is the Hata model [4] based on Okumura's data which has standard deviation between predicted and measured path loss value about 10 to $14 \mathrm{~dB}$. The disadvantages of the models include those that do not reflect the rapid terrain variations and could not be used in small cells. Walfisch-Bertoni model includes the impact of rooftop and building height in the path loss models for urban [5]. The performance of some VHF/UHF propagation models is analysed in the presence of irregular terrain covered by forest. The comparisons are made between the model recommended by ITU-R and a measurement survey carried out in Rio de Janeiro City. In addition, predictions generated using a two-dimensional (2D) ray tracing method were incorporated into the analysis [6]. Ray tracing-based models compute radio ray propagation accounting correctly for the reflection, diffraction, and scattering [7]. Ray tracing method has an advantage over traditional prediction methods: accurate site-specific field strength prediction. The analysis of outdoor microcellular radio propagation characteristics based on the method of shooting and bouncing ray tracing (SBR)/image is proposed in [8], and the combination of the Ray tracing and the empirical model for path loss prediction is analysed in [9]. Nevertheless, the techniques have some weaknesses: they are unreliable under ducting conditions, suffering difficulties when transiting from one type of region/cell to another, and when handling the backscattering problem, they require field input on the building surfaces which is difficult to obtain within simple assumptions. The parabolic equation (PE) approximation to the wave equation is a full-wave method, combining terrain diffraction, atmospheric ducting, and great flexibility in the specification of the building geometry and electrical parameters, thus accounting simultaneously 
and accurately for the diffraction, refraction, and scattering $[10,11]$. The performance of mobile radio systems is highly dependent on the fading phenomena, the fast fading results from the superposition of randomly scattered multipath replicas. The statistical properties of fast fading have been investigated extensively in the literature [12]. The slow fading occurs mainly due to the effects of buildings and terrain features and determines variations of the local mean. The autocorrelation behaviour of the slow fading is important in the mobile communication technologies such as the design of handover scheme [13]. Based on the measurement data in urban micro and suburban macro environments, a simple exponential model has been proposed in [14] for the autocorrelation function (ACF). Recently, based on the measured data in urban macro environments, which are a mixture of line-of-sight (LOS) and non-line-of-sight (NLOS) conditions but mostly NLOS, the exponential decaying sinusoid (EDS) model has been proposed in [15] for empirical ACFs of individual routes. The measurement of path loss and shadowing correlation on two TV channels are performed in urban, in which the exponent and autoregressive model for correlation function are presented [16].

To address the path loss and large-scale fading, in this paper, the wavelet method is used to study the propagation loss and slow fading model. For wave propagation in urban, the environments are very complex and changed rapidly from place to place. The wavelet transforms are based on small wavelets with limited duration. The translated-version wavelets locate where we concerned. The scaled-version wavelets allow us to analyse the signal in different scales. The measurement is performed in Beijing City where the large buildings are crowed. The measurement data are analysed using wavelet transform method, and the results show that the path loss and slow fading can be obtained on different scales, which give us more information to the radio system design and optimization.

\section{Optimal Wavelet Selection}

A wavelet is a wave-like oscillation with an amplitude that begins at zero, increases, and then decreases back to zero. The wavelets can be used to extract information from many different kinds of data; in addition, the sets of wavelets are generally needed to analyse data fully. The discrete wavelet transforms use the discrete shift and scale parameters.

From the view of multiresolution, a subset of functions in the $L^{2}(R)$ space can be expanded in terms of only scaling functions and is expressed as

$$
f(x)=\sum_{k=-\infty}^{\infty} c_{k} \phi(x-k)
$$

A two-dimensional family of functions is generated by scaling and translating the original scaling function $\phi(x)$ by

$$
\phi_{j, k}=2^{j / 2} \phi\left(2^{j} x-k\right)
$$

If we fix $j=0$ and only vary $k$, then we have a small subspace $V_{0}=\left\{\phi_{0, k}\right\}$. However, if we are allowed to have $j=1$, then the subspace $V_{1}=\left\{\phi_{1, k}\right\}$ will have more member than space $V_{0}$. Similarly, the subspace $V_{2}$ have more member than $V_{1}$. To extend this argument, a nesting of the spanned spaces is obtained as

$$
\cdots V_{-2} \subset V_{-1} \subset V_{0} \subset V_{1} \subset V_{2} \subset \cdots \subset L^{2}(\mathrm{R}) .
$$

The difference between subspace $V_{0}$ and $V_{1}$ can be defined with the wavelet subspace $W_{1}$ in the form

$$
V_{j}=V_{j-1} \oplus W_{j+1} \text {, }
$$

where $\oplus$ is the direct sum of two subspaces. It follows that

$$
L^{2}(R)=V_{0} \oplus W_{0} \oplus W_{1} \oplus W_{2} \oplus \cdots
$$

The argument in (5) provides the theoretical justification for an expansion of the function $f(x)$ in the space $L^{2}(R)$.

$$
f(x)=\sum_{k=-\infty}^{\infty} c_{k} \phi(x-k)+\sum_{k=\infty}^{\infty} \sum_{j=0}^{\infty} d_{j, k} \psi\left(2^{i} x-k\right) .
$$

The selection of suitable mother wavelet basis function is necessary for efficient analysis of propagation loss data. Optimal wavelet will lead to the maximization of coefficient values and will produce the highest local maxima of the data. The best characterization of scale content of propagation loss signal is possible with an optimally selected wavelet filter. The daubechies wavelet picks up the minute detail which is missed by the other wavelet algorithm. Even if a signal is not represented well by one member of the daubechies family, it may be efficiently represented by another [17]. The daubechies wavelets are widely used in solving a broad range of problems, e.g., self-similarity properties of a signal and signal discontinuities. In general, the daubechies wavelets are chosen to have the highest number $N$ of vanishing moments, which is directly related to the number of coefficients. So the trade-off is taken between the vanishing moments and the efficiency. In the analysis of the wave propagation, the $\mathrm{db} 7$ is taken as the wavelet basis because the basis can give the required smoothing and efficiency. The lower vanish moment basis such as $\mathrm{db} 1$ to $\mathrm{db} 6$ is not smoothing as $\mathrm{db} 7$, and the higher vanishing moment basis has less efficiency compared to db7. The low-pass and high-pass decomposition and reconstruction filter banks of $\mathrm{db} 7$ are shown as Figure 1.

The choice of the decomposition level is one of the key factors for the wavelet decomposition. The results affirmed that the wavelet-based modeling performance is sensitive to the choice of the decomposition level, which is determined by the data analysed. Most studies usually choose the decomposition level according to data length, in which the level is determined as $L=\log (\mathrm{ND})$, where ND is the data length. In this paper, the choice of the decomposition level is depended to the smoothness of the space range. For the 

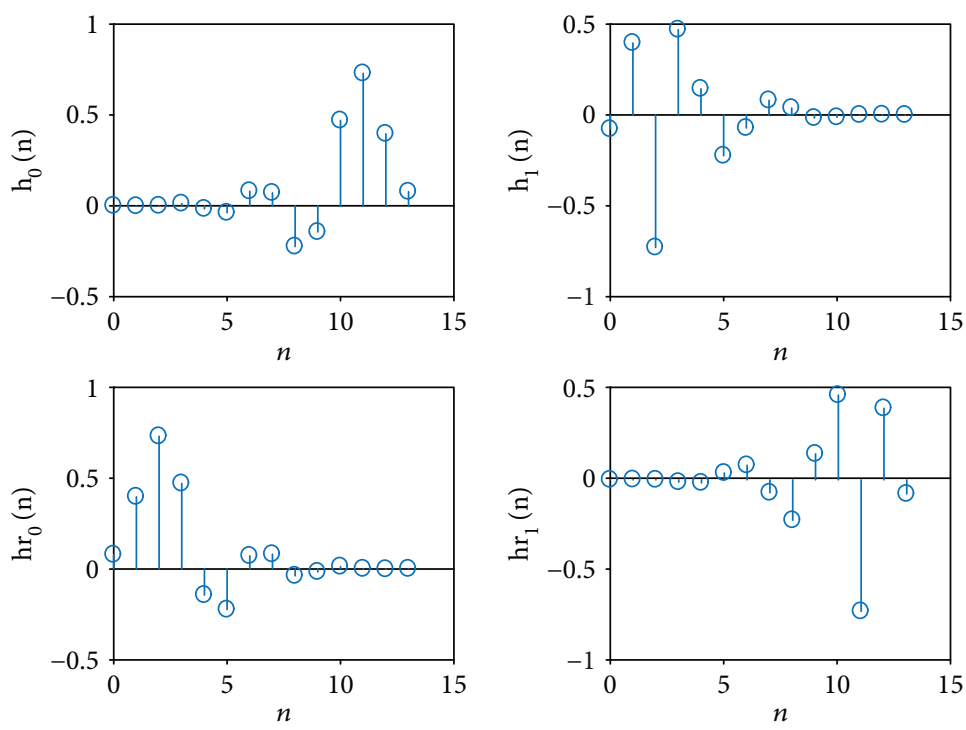

Figure 1: The filter banks of wavelet basis db7. The $h_{0}(n)$ and $h_{1}(n)$ are low-pass and high-pass decomposition filters, respectively. The $h r_{0}(n)$ and $h r_{1}(n)$ are low-pass and high-pass reconstruction filters, respectively.

large-scale propagation loss, the linear property is required that is compared with the Hata model and the decomposition level is selected as 11, in which the spanned space range is about 2048 sample points. For the large-scale fading correlation, the selection of the decomposition level is determined according concerned space range.

The propagation data measured at the Beijing City of China are used. Their arithmetic average values in each 100-meter range are used as basis data for modeling. The signal intensity values are transformed to the propagation loss values. The basic idea in the analysis of wavelet is that the irregularity of propagation loss is caused by the random distribution building. With the wavelet scaling basis function, the irregularity can be smoothed and the smoothness depends on the scale range. The algorithm for the analysis is as follows. Firstly, the preprocessed propagation loss data is as the initial data,

$$
x_{0}(n)=x(n)
$$

Second, the low-pass filter $h_{0}(n)$ and high-pass filter $h_{1}(n)$ are used to get the decomposed average signal $x_{m}(n)$ and detail signal $d_{m}(n)$ at level $m$.

$$
\begin{aligned}
& x_{m}(n)=\sum_{i} x_{m-1}(i) \cdot h_{0}(2 n-i), \\
& d_{m}(n)=\sum_{i} x_{m-1}(i) \cdot h_{1}(2 n-i) .
\end{aligned}
$$

For the inverse of wavelet transform, the smoothing is performed by ignoring the detail at each step.

$$
\tilde{x}_{m-1}(n)=\sum_{i} h_{0}(2 i-n) \cdot x_{m}(i) .
$$

Finally, the smoothing propagation loss at scale $m$ is obtained by

$$
\tilde{x}(n)=\tilde{x}_{0}(n)
$$

The obtained reconstructed propagation loss signal gives a smoothed propagation loss at that scale, which can weaken the effects of the irregularity caused in the scale range.

\section{Measurement and Data Preprocessing}

The radio wave propagation measurements have been performed at $546 \mathrm{MHz}$; the wavelength is $0.56 \mathrm{~m}$, in the urban environment of Beijing, China. The height of transmitter antenna is 293 meters, and the gain of antenna is $9.5 \mathrm{~dB}$. The receiver, which is controlled by a laptop PC, is equipped with a global positioning system (GPS) and an Omnidirectional antenna at the height of $1.8 \mathrm{~m}$ above ground. The collected test data have been originated from the measurement campaign performed in west part urban of Beijing along a wide variety of measurement paths around the transmitter on the TV tower. The data of electric field at 6767 points are collected.

In order to get the propagation loss and fading characteristics, the test data should be processed for the later analysis. Owing that the distribution of the test points is random, the data are firstly ordered according the distance from the transmitter and then the data on same distance from the transmitter are averaged to keep the unique value at the position. Finally, the interpolation is used to obtain the data at the equal increment along the distance from the transmitter. Figure 2 shows the original test data and the interpolated data, and the difference is small. The interpolated data is used as the basis for the propagation loss and slow fading for the study of the wireless channel. 


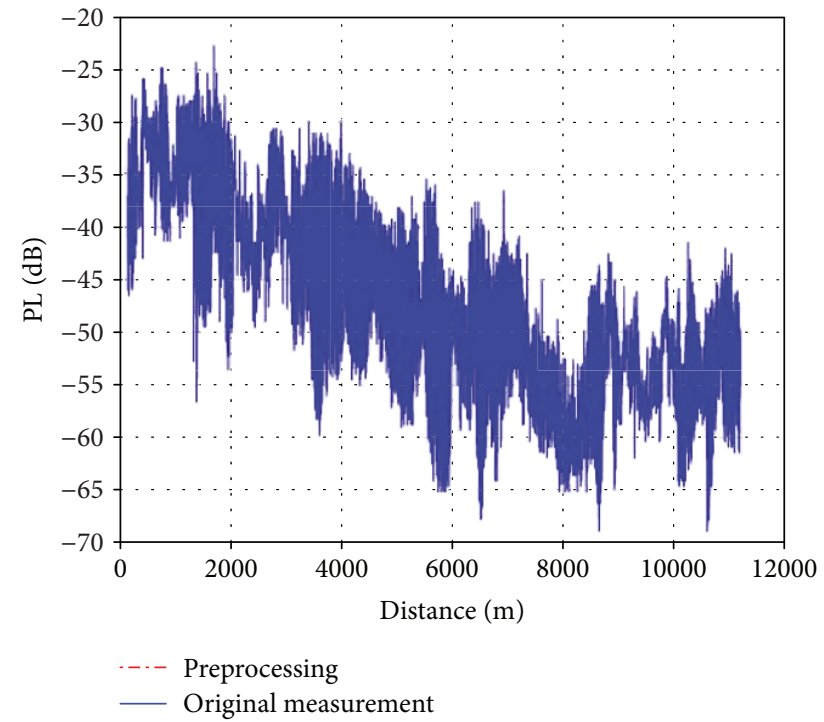

FIGURE 2: The test data and the interpolated data.

\section{Analysis of Propagation Loss}

For the purpose of comparison, the optimized Hata model is use. The Hata model has the expression:

$$
\mathrm{PL}=a+b \cdot \log (R)=a+b \cdot x
$$

The condition of the best of the theoretical model curve with a given set of experiment data would be met if the function of sum of deviation squares is minimum and is denoted as

$$
F(a, b)=\sum_{i=1}^{n}\left|y_{i}-\operatorname{PL}\left(x_{i}, a, b\right)\right|^{2}=\min ,
$$

where $y_{i}$ is measurement result at the distance $x_{i}$ and $a$ and $b$ are parameters on optimization. All partial differentials of $F(a, b)$ should be equal to zero:

$$
\begin{aligned}
& \frac{\partial F}{\partial a}=0, \\
& \frac{\partial F}{\partial b}=0 .
\end{aligned}
$$

The solution of (13) may be expressed as

$$
\begin{aligned}
& \sum\left(y_{i}-\mathrm{PL}\left(x_{i}, a, b\right)\right) \frac{\partial \mathrm{PL}}{\partial a}=\sum\left(y_{i}-a-b x_{i}\right) * 1=0 \\
& \sum\left(y_{i}-\mathrm{PL}\left(x_{i}, a, b\right)\right) \frac{\partial \mathrm{PL}}{\partial b}=\sum\left(y_{i}-a-b x_{i}\right) * x_{i}=0 .
\end{aligned}
$$

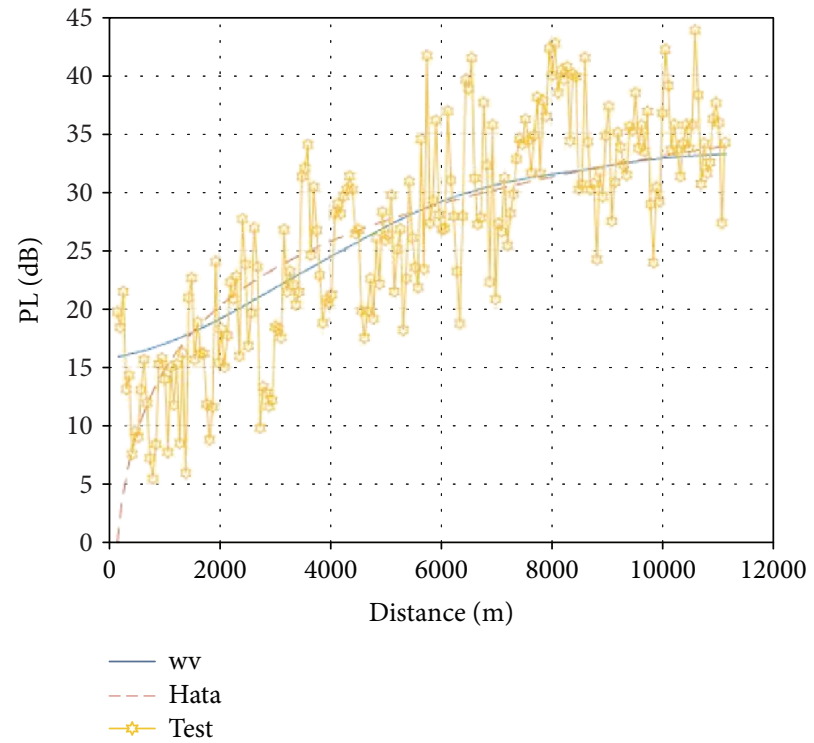

FIgURE 3: Propagation loss versus distance. wv: wavelet method using $\mathrm{db} 7$ and level 11; Hata: optimized Hata model; test: measurement data.

By rearrange of elements in (14), the following expression can be obtained:

$$
\begin{aligned}
& a=\frac{\sum_{i} x_{i}^{2} \sum_{i} y_{i}-\sum_{i} x_{i} \sum_{i} x_{i} y_{i}}{n \sum_{i} x_{i}^{2}-\left(\sum x_{i}\right)^{2}} \\
& b=\frac{n \sum_{i} x_{i} y_{i}-\sum_{i} x_{i} \sum_{i} y_{i}}{n \sum_{i} x_{i}^{2}-\left(\sum x_{i}\right)^{2}}
\end{aligned}
$$

The propagation loss in the west part of Beijing is shown in Figure 3. In order to display clearly, the measured data are used by every other 20 points, which are displayed as "o" marks. The propagation loss computed with the wavelet method for whole path is expressed as a solid line, which use the low frequency approximation level $N=11$, among the average 2048 sample data. The optimized Hata model is shown as the dashed line, in which optimized parameters $a=-35.8$ and $b=-18.3$. From the figure, it can be seen that the large decomposition level is more closed to the optimized Hata model, owing that the large decomposition has more sample data to smooth. The advantage of the wavelet method is its multiscale for the problem of wave propagation. Considering the variation of urban path from place to place, a simple Hata model cannot account to the changes from place to place. With the wavelet method, the propagation loss on different scales can be obtained, which enlightens us with the variation of propagation loss along as propagation distance and reduces the prediction bias. The propagation loss in level $N=6$ is shown in Figure 4 . It can be seen that the propagation loss in level 6 can catch up the change trend of the measurement data that may be caused by the building with different scale sizes. The average distance increment is 2.7 meters for the analyzed data. For level 11 in 


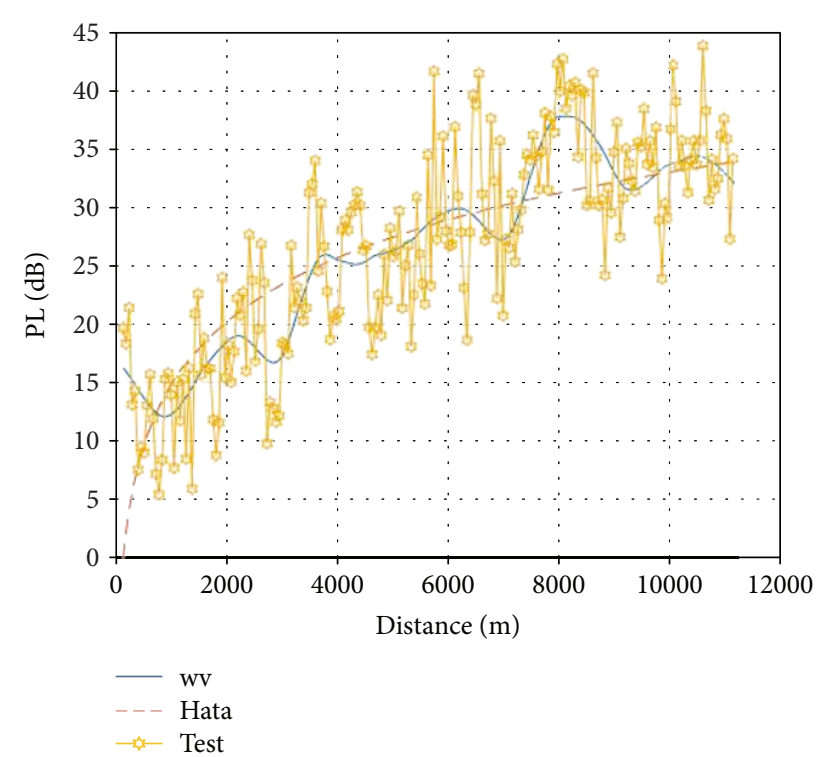

FIgURe 4: Propagation loss versus distance. wv: wavelet method using $\mathrm{db} 7$ and level 6; Hata: optimized Hata model; test: measurement data.

the wavelet method, the data average is performed on 2048 samples' position, which spans about 5529.6 meters and can explain why the propagation loss curve is more closed to the Hata optimized model. For level 6, the average is on the 64 sample data that span the distance of 172.8 meters. Therefore, the propagation loss shows the average on the scale 172.8 meters, which is the approximate to the measurement data more closely.

\section{Slow Fading and Correlation}

For the wireless network planning, the understanding of the fading in the different scale is very important. In many studies which investigate correlation between spatially distributed antennas, a few assumptions or settings are utilized. The normalized autocovariance of zero-mean shadowing $\eta$ in decibels is expressed as

$$
\rho\left(\delta_{m}\right)=\frac{\sum_{k} \eta\left(d_{k}\right) \eta\left(d_{k}+\delta_{m}\right)}{\sum_{k} \eta^{2}\left(d_{k}\right)}
$$

The correlation distance $\delta_{m}$ is used in [16]. A common method to represent correlated shadowing is by means of a simple empirical model with a single parameter, which assumes the exponential decay of correlation with distance. The correlation distance parameter is obtained by

$$
\sum_{m}\left|\rho\left(\delta_{m}\right)-\exp \left(-\frac{\delta_{m}}{\Delta_{c}}\right)\right|^{2}=\min .
$$

In this study, the fading correlation is studied by a wavelet method. From wavelet theory, different scale functions can give us the information about the average in that scale. Moreover, the fading correlation is related with the

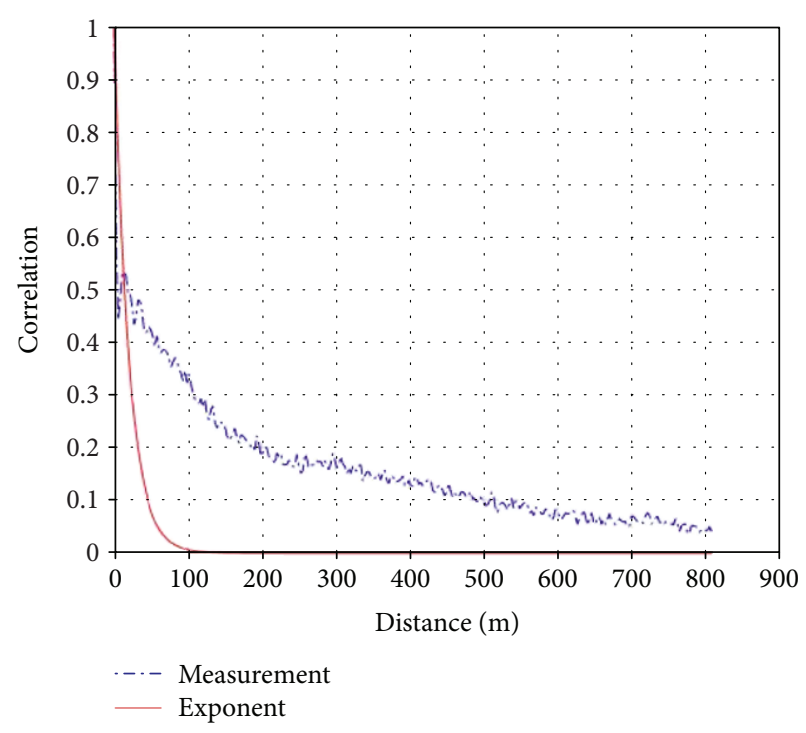

FIGURE 5: Slow fading correlation for measurement data. The fading correlation distance is 13.8 meters for the measurement data.

propagation loss in the scale. Daubechies wavelets are widely used in solving a broad range of problems, e.g., self-similarity properties of a signal. The different scale has a different support range, and the support width of the wavelet is $2 L-1$. The scaling function represents the lower frequency part in the wavelet expansion, and the wavelet function represents the higher frequency part. The loss function on scale $J$ can be represented as

$$
L_{J}(x)=\sum_{k=1}^{N} c_{k}^{J} \phi_{J, k}(x)
$$

where $\phi_{J, k}(x)$ is a scaling function in scale $J$. For the scale $J$, the support width is $(2 \times L-1) \times 2.7$ meters because the distance increment of the sample data is $2.7 \mathrm{~m}$. The selection scale $J$ can give us the information about local trend in the span of the space range. For the scale $L=2$, the span of the space range is about $8.1 \mathrm{~m}$. The propagation loss function represents the variation in that range, and the correlation distance will be short because the loss function has more fluctuation as distance change. If the scale level is increased, the loss function in more large space range is average and the loss function will have a more slow fluctuation. The correlation distance will be increased because of the slow fluctuation in loss function. To find the fading correlation, the measurement data of zero mean is obtained by subtracting the measurement data with optimized Hata data. Moreover, the correlation function of the measured data using the conventional exponent correlation method in [14] is computed and shown in Figure 5. It can be seen that the fading correlation distance is very short and is about $13.8 \mathrm{~m}$.

The correlation function using the wavelet method is also computed and shown in Figure 6. In order to fit the exponent correlation function, the scale level 2 is selected to compute the loss function and the fading correlation distance is about 152.5 meters. It can be seen that the computed correlation 


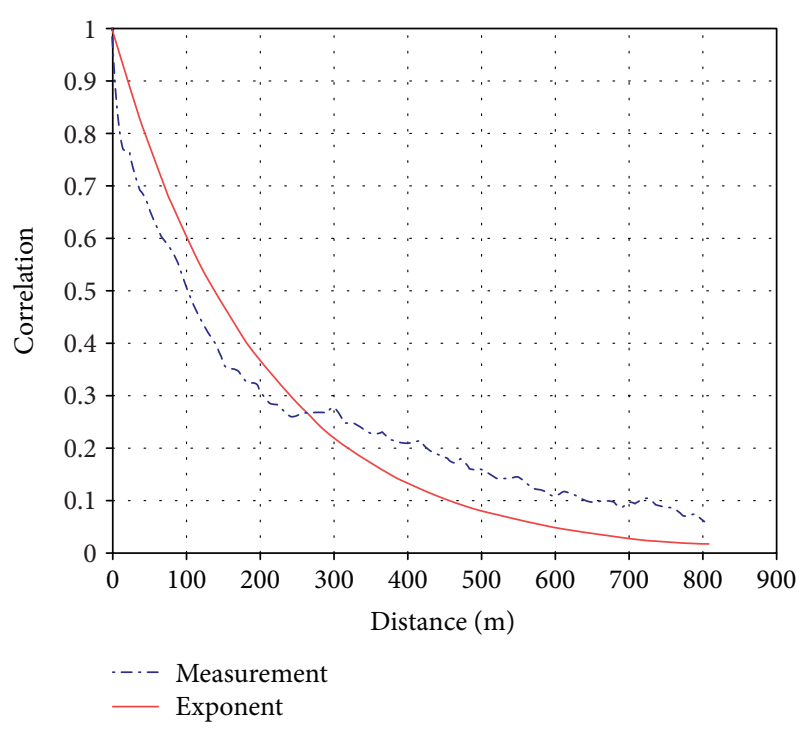

Figure 6: Slow fading correlation using scale 2 wavelet. The fading correlation distance is 152.5 meters for the wavelet method level 2 .

function has a good agreement with the exponent correlation function. For the level 3, the similar results are obtained. The simulations are also carried out to the other levels of wavelet, and the results show that the larger scale has large correlation distance.

\section{Conclusion}

Wavelet analysis is capable of revealing aspects of data such as trends which other signal processing methods are neglecting. One major advantage afforded by wavelets is the ability to perform local analysis. The wavelet basis selection is an important step in the analysis. Daubechies wavelets are used in analyzing the large-scale correlation problems. The choice of the decomposition level is one of the key factors for the wavelet decomposition. For the large-scale propagation loss, the linear property is required that is compared with the Hata model and the decomposition level is selected as 11, in which the spanned space range is about 2048 sample points. For the large-scale correlation, the selection of the decomposition level is determined according with concerned space range.

A wavelet method is proposed to evaluate the modeling of the first- and second-order statistics of large-scale fading from the signal strength measurement. The experiment evaluation is performed in the west part of Beijing which has many high buildings with high density, by sampling digital TV signal on channel DS-22546 MHz.

By wavelet method, the path loss is calculated and the comparison with an optimized Hata model is evaluated. At large scale, two methods show the similar result to the path loss. However, the different scale path loss can be obtained with a wavelet method, which can describe the change of the average path loss from place to place. From the empirical model with second-order statistics, correlation distance is about 13.8 meters, which is computed from measurement data. By wavelet scale 2, the correlation distance is about 152.5 meters. The other simulation shows that the correlation distance of the large-scale fading is related to the spatial scale.

\section{Data Availability}

The measured data used to support the findings of this study have not been made available because it belongs to the Academy of Broadcasting Planning SARFT, Beijing, China.

\section{Conflicts of Interest}

The authors declare that there are no conflicts of interest regarding the publication of this paper.

\section{References}

[1] M. Zorzi, R. R. Rao, and L. B. Milstein, "ARQ error control for fading mobile radio channels," IEEE Transactions on Vehicular Technology, vol. 46, no. 2, pp. 445-455, 1997.

[2] M. Simon and M. Alouini, Digital Communication over Fading Channels, Wiley-IEEE Press, 2006.

[3] ITU-R, Recommendation ITU-R P.1546-5: Method for pointto-area predictions for terrestrial services in the frequency range $30 \mathrm{MHz}$ to $3000 \mathrm{MHz}$., 2013.

[4] M. Hata, "Empirical formula for propagation loss in land mobile radio services," IEEE Transactions on Vehicular Technology, vol. 29, no. 3, pp. 317-325, 1980.

[5] H. Bertoni, Radio Propagation for Modern Wireless Systems, Prentice Hall Professional Technical Reference, 2000.

[6] F. M. da Costa, L. A. R. Ramirez, and M. H. C. Dias, "Analysis of ITU-R VHF/UHF propagation prediction methods performance on irregular terrains covered by forest," IET Microwaves, Antennas \& Propagation, vol. 12, no. 8, pp. 1450-1455, 2018.

[7] T. K. Sarkar, Zhong Ji, Kyungjung Kim, A. Medouri, and M. Salazar-Palma, "A survey of various propagation models for mobile communication," IEEE Antennas and Propagation Magazine, vol. 45, no. 3, pp. 51-82, 2003.

[8] X. Ma, Y. Liu, Q. Shi, and Y. Zhang, "Simulation and analysis of outdoor microcellular radio propagation characteristics based on the method of SBR/image," Journal of Computer and Communications, vol. 3, no. 3, pp. 86-92, 2015.

[9] A. Bhuvaneshwari, R. Hemalatha, and T. Satyasavithri, "Semi deterministic hybrid model for path loss prediction improvement," Procedia Computer Science, vol. 92, pp. 336-344, 2016.

[10] C. A. Zelley and C. C. Constantinou, "A three-dimensional parabolic equation applied to VHF/UHF propagation over irregular terrain," IEEE Transactions on Antennas and Propagation, vol. 47, no. 10, pp. 1586-1596, 1999.

[11] D. I. Sirkova and M. A. Mikhalev, "A review of the applicability of the main EM field calculation and assessment models in wireless communications design," in Proceedings of ICEST02, pp. 225-228, Nis, Yugoslavia, 2002.

[12] L. Barclay, Propagation of Radiowaves, The Institution of Engineering and Technology, London, United Kingdom, 3rd edition, 2013.

[13] W. Kim, H. Lee, J. J. Park, M.-D. Kim, and H. K. Chung, "An autocorrelation model for shadow fading in rural macro environments," in 2012 18th Asia-Pacific Conference on Communications (APCC), pp. 323-326, Jeju Island, South Korea, October 2012. 
[14] M. Gudmundson, "Correlation model for shadow fading in mobile radio systems," Electronics Letters, vol. 27, no. 23, pp. 2145-2146, 1991.

[15] Y. Zhang, J. Zhang, D. Dong, X. Nie, G. Liu, and P. Zhang, “A novel spatial autocorrelation model of shadow fading in urban macro environments," in IEEE GLOBECOM 2008 - 2008 IEEE Global Telecommunications Conference, pp. 1-5, New Orleans, LA, USA, 2008.

[16] H. Kremo, "Characterization of first and second order statistical of large scale fading using vehicular sensors," in Vehicular Technology Conference (VTC Spring), 2015 IEEE 81st, pp. 1-5, Glasgow, UK, 2015.

[17] S. Mehrotra, "Analysis of ECG with db10 wavelet using Veri$\log$ HDLL," International Journal of Technical Research and Applications, vol. 3, no. 3, pp. 142-146, 2015. 


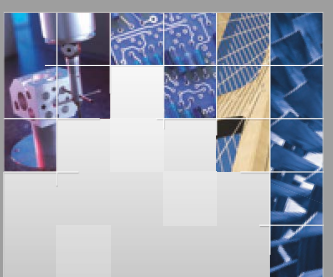

\section{Enfincering}
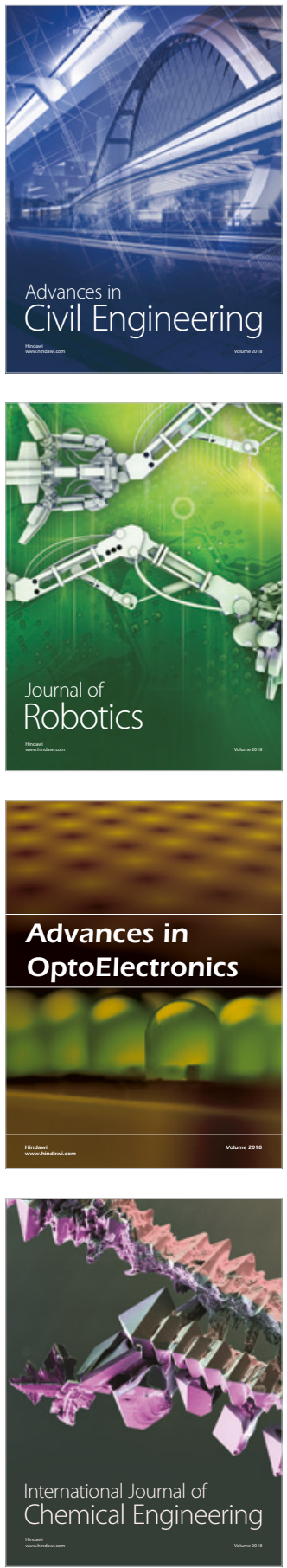

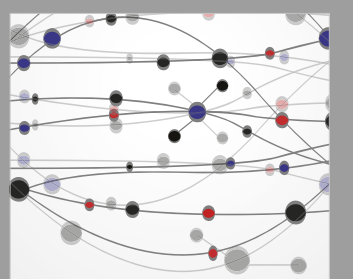

\section{Rotating \\ Machinery}

The Scientific World Journal

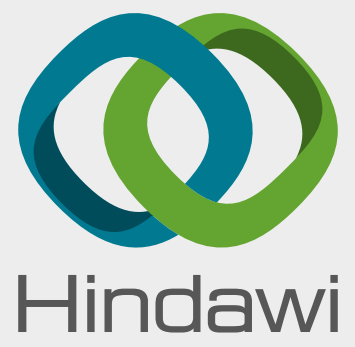

Submit your manuscripts at

www.hindawi.com
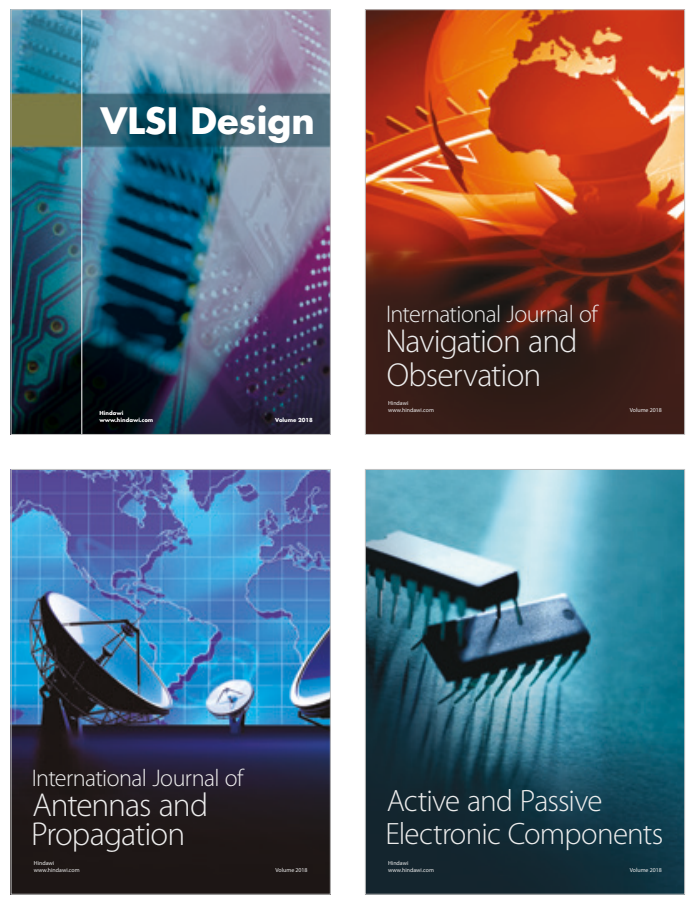
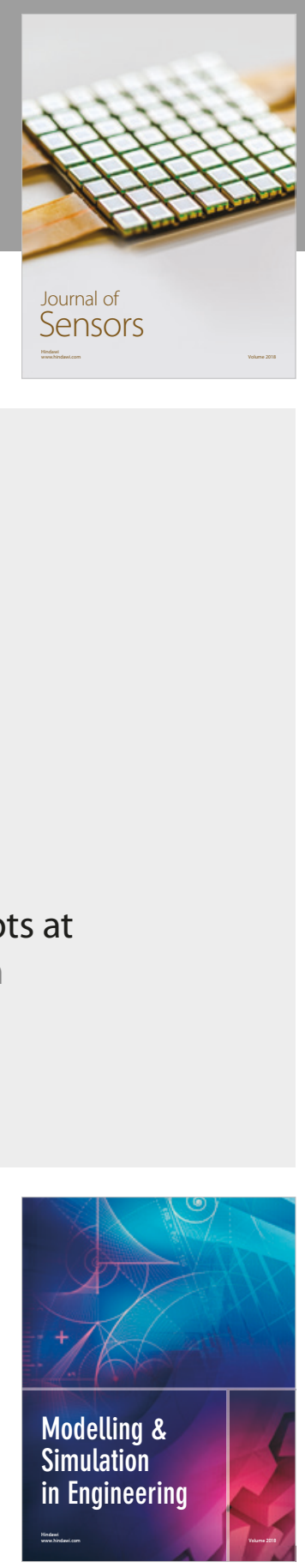

\section{Advances \\ Multimedia}
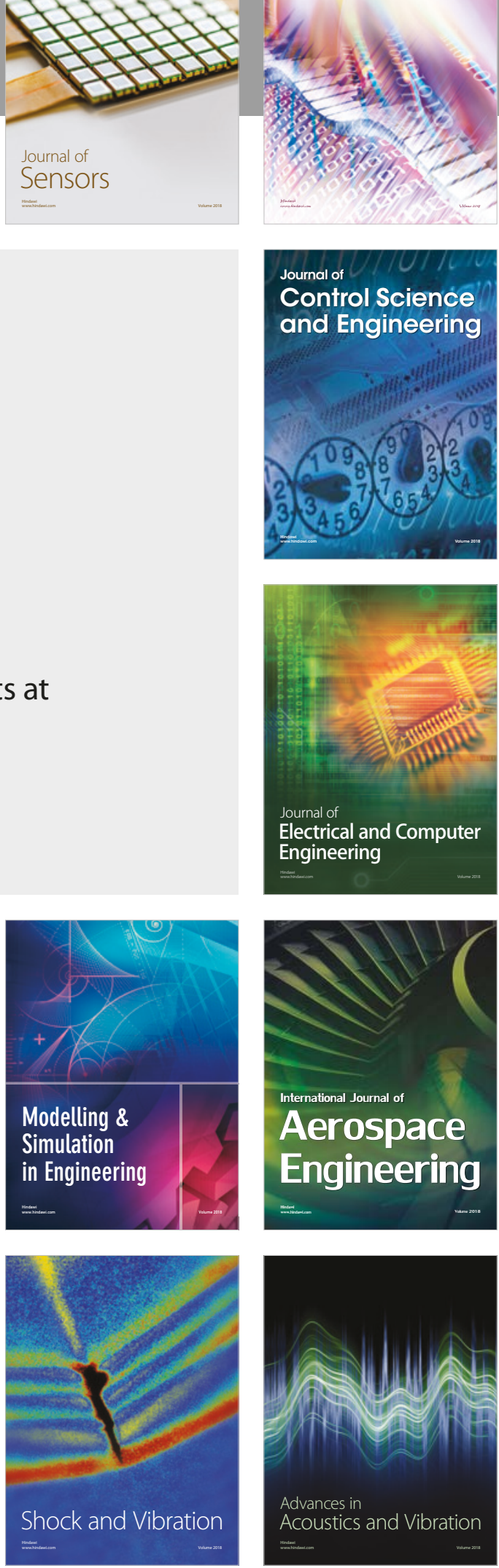